THE Astrophysical JouRnal, 477:424-430, 1997 March 1

(C) 1997. The American Astronomical Society. All rights reserved. Printed in U.S.A.

\title{
X-RAY OBSERVATIONS OF X2127+119/AC 211 IN THE METAL-POOR GLOBULAR CLUSTER M15: AN X-RAY MEASURE OF METALLICITY?
}

\author{
D. J. CHRISTIAN \\ Center for EUV Astrophysics, 2150 Kittredge Street, University of California, Berkeley, CA 94720-5030; damian@cea.berkeley.edu \\ AND \\ A. P. Smale, J. H. Swank, and P. J. Serlemitsos \\ NASA/Goddard Space Flight Center, Code 666.0, Greenbelt MD, 20771; alan@osiris.gsfc.nasa.gov, swank@pcasun1.gsfc.nasa.gov, \\ peter@heasfs.gsfc.nasa.gov \\ Received 1996 March 27; accepted 1996 September 24
}

\begin{abstract}
We present results from observations of the $17.1 \mathrm{hr}$ low-mass X-ray binary X2127+119 (AC 211) in M15 obtained with the broadband X-ray telescope (BBXRT) in 1990 December and observations obtained with the solid state spectrometer (SSS) together with the monitor proportional counter (MPC) on the Einstein Observatory in 1978 and 1979. The continuum can be well described with the twocomponent model of a blackbody $(k T \sim 1 \mathrm{keV})$ and a power law that was found to fit the EXOSAT medium-energy data. Short-term variability observed by the SSS and MPC was related to changes in the intensities of both components in this description. The SSS and MPC observed factor of 10 differences in column density in observations separated by a few binary orbits. These observations confirm correlations between the steepness of the spectrum and the column density, and column density with phase. The percentage contribution of the blackbody is anticorrelated with the factor of 4 variations in the apparent luminosity, but other correlations are weak. A $1000 \mathrm{~s}$ observation by BBXRT at photometric phase 0.9 provides an upper limit of $\sim 150 \mathrm{eV}$ to narrow Fe $\mathrm{K}$ line emission. BBXRT spectra are generally inconsistent with the overall cluster metallicity of $1 \%$ of solar, although complex spectral models can be more consistent with the cluster metallicity. A model separating the absorption of $\mathrm{H}$ and $\mathrm{He}$ from the metals gives $1.25_{-0.2}^{+0.03} \times 10^{18} \mathrm{~cm}^{-2}$ for the column density of metals in the absorbing gas associated with the binary system. The noted correlations between spectral parameters, luminosity, and their relation to orbital phase are consistent with a disk plus moderate-sized corona interpretation.
\end{abstract}

Subject headings: binaries: close - globular clusters: individual (M15) - stars: abundances stars: individual $(\mathrm{X} 2127+119)-\mathrm{X}$-rays: stars

\section{INTRODUCTION}

The X-ray source X2127+119, located near the center of the globular cluster M15 and coincident with the 15th magnitude optical star AC 211 (Aurière, Le Fèvre, \& Terzan 1984), has been extensively studied because of its complex optical and X-ray behavior. However, classifying $\mathrm{X} 2127+119$ as a type of low-mass X-ray binary (LMXB) and understanding its geometry has been made difficult by this diversity. Optical modulations are 3-7 times greater than those at X-ray energies, and are the largest seen from any X-ray binary. The folded light curve of X2127+119 shows a broad optical minimum at phase 0.0 , but with extremely erratic and complex X-ray and optical behavior between phases 0.2 and 0.7. A $17.11 \mathrm{hr}$ optical period has been reported (Ilovaisky et al. 1993), which is twice as long as previously believed. Modulations in X-ray observations by HEAO 1, EXOSAT, and Ginga have been shown to be consistent with this new period, as well as the older shorter period. The low $L_{\mathrm{X}} / L_{\mathrm{opt}}$ ratio for $\mathrm{X} 2127+119$, typical of accretion disk corona (ADC) sources, and optical spectroscopy, showing Balmer lines with P Cygni profiles and He II and $\mathrm{H} \alpha$ emission, support the idea that an extended corona or wind above the accretion disk is present. However, the "classical ADC" idea was shaken by detection of a very luminous X-ray burst by Ginga (Dotani et al. 1990; van Paradijs et al. 1990). The burst result did establish that the compact object must be a neutron star and that we can see the compact object directly, but alternative explanations are needed to explain the observed X-ray and optical behavior.
We present results of simultaneous observations by the Einstein solid state spectrometer (SSS; $0.5-4.5 \mathrm{keV})$ and monitor proportional counter (MPC; $1.2-20.0 \mathrm{keV}$ ), and two short observations obtained by the broadband X-ray telescope (BBXRT; 0.3-12.0 keV). Our Einstein observations of $\mathrm{X} 2127+117$ sample several photometric phases with notable changes in spectral parameters, especially an order-of-magnitude increase in column density near phase 0.5 . The moderate-resolution BBXRT observations provide $\sim 150 \mathrm{eV}$ upper limits to narrow $\mathrm{Fe} \mathrm{K}$ emission between 6.4 and $6.7 \mathrm{keV}$ and suggest a nearly solar composition of metals for this source. We present a summary of the observations and analysis in $\S 2$ of this paper. In $\S 3$ we present the spectral results, including BBXRT observations to determine the X-ray metallicity (\$3.2) and upper limits to emission lines (\$3.3). Implications of the results in a physical context are discussed in $\S 4$.

\section{OBSERVATIONS AND ANALYSIS}

The Einstein Observatory SSS and MPC simultaneously observed X2127+119 between 1978 December and 1979 June (1978 January 0.0= JD 2,443,508.5). The Einstein Observatory is described by Giacconi et al. (1979). The SSS is described in detail by Joyce et al. (1978), and the MPC by Gaillardetz et al. (1978). Typical observation lengths were $10,000 \mathrm{~s}$ with 3000 to $4000 \mathrm{~s}$ of good source data (excluding Earth occultations and South Atlantic Anomaly passages). The BBXRT (Weaver et al. 1995) observed X2127+119 on 1990 December 8 and accumulated nearly $1 \mathrm{ks}$ of good data 
near phase 0.9 using the ephemeris of Ilovaisky et al. (1993). A second observation of X2127+119 on 1990 December 9 only obtained $200 \mathrm{~s}$ of usable data because of poor pointing.

The observation dates, count rates, and accumulation times for the Einstein and BBXRT observations are presented in Tables 1 and 2, respectively. The SSS observation of 1978 December 5 has a very high ice thickness covering the detector. The 1979 May and June observations have moderate ice thickness. For this reason observed SSS count rates are not indicative of the true source flux.

The SSS PHA (0.5-4.5 keV) and the MPC (1.2-20.0 keV) temporal data were accumulated in $2.56 \mathrm{~s}$ bins using $H E A O$ 2 software. SSS and MPC light curves are shown in Figures $1 a$ and $1 b$. Phases for the $17.1 \mathrm{hr}$ period derived from both optical and X-ray data are indicated (Ilovaisky et al. 1993). Source variability superseded the ability to distinguish the periodicity in a folded light curve. In most cases the MPC accumulation time is slightly larger than the SSS accumulation time, because the SSS was sensitive to fluorescence of the Earth's atmosphere by solar flux, and data for which the line of sight grazed the bright Earth were rejected as indicated by a high OPTO firing rate, which indicates how often the field effect transistors were discharged by the LED (Joyce et al. 1978).

\subsection{Temporal}

Root mean square fluctuations were studied on short timescales in several energy bands. Root mean square values for a $40.96 \mathrm{~s}$ timescale, chosen to maximize counting statistics, in the SSS energy bands of $0.5-1.2 \mathrm{keV}$ were $22 \%$ for the day 495 observation with 50\% upper limits for other observations. The SSS $1.2-3.5 \mathrm{keV}$ bandpass had rms varia-

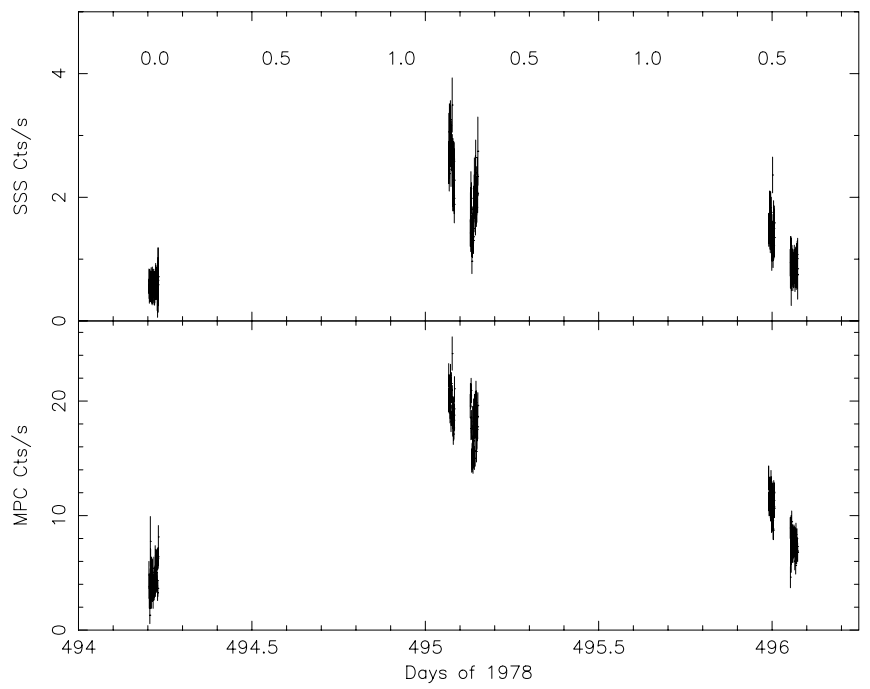

FIG. 1.-SSS (0.5-4.5 keV) and MPC (1.2-20 keV) 1979 May 9, 10, and 11 light curves plotted as a function of days of 1978. Phases from the 17.1 hr ephemeris of Ilovaisky et al. (1993) are indicated at the top.

bility of $20 \%-30 \%$. MPC results for the $1.2-3.5,3.5-10$, and $10-20 \mathrm{keV}$ bands were $19 \%-34 \%, 14 \%-24 \%$, and $<50 \%$, respectively. The BBXRT $0.6-10.0 \mathrm{keV}$ light curve had a $3 \sigma$ upper limit to rms variability of $5 \%$ on 40 s timescales.

\subsection{Spectral}

SSS spectra were accumulated in $2.56 \mathrm{~s}$ bins for each spacecraft orbit or for time intervals of constant source intensity, except for observations on May 10 and 11, in

TABLE 1

Einstein OBSERVATION LOG

\begin{tabular}{|c|c|c|c|c|}
\hline \multirow[b]{2}{*}{ OBSERVATION $^{\mathrm{a}}$} & \multicolumn{2}{|c|}{ SSS } & \multicolumn{2}{|c|}{ MPC } \\
\hline & Counts $\mathrm{s}^{-1}$ & Live Time (s) & Counts s ${ }^{-1}$ & Live Time (s) \\
\hline $1978 \operatorname{Dec} 5(339) .$. & 1.7 & 5737 & 12.6 & 6851 \\
\hline 1979 May 9 (494) . & 0.6 & 1661 & 4.4 & 2422 \\
\hline 1979 May $10(495) \ldots \ldots$ & 2.4 & 3082 & 18.3 & 3372 \\
\hline 1979 May $11(496) \ldots \ldots$. & 1.3 & 2897 & 8.9 & 3438 \\
\hline 1979 Jun $2(518) \ldots \ldots \ldots$ & 1.0 & 3656 & 7.9 & 7309 \\
\hline
\end{tabular}

${ }^{a}$ Days of 1978 shown in parentheses. 1978 Jan $0=$ JD 2,443,508.5.

TABLE 2

BBXRT OBSERVATION LOG

\begin{tabular}{|c|c|c|c|c|}
\hline \multirow[b]{2}{*}{ OBSERVATION $^{\mathrm{a}}$} & \multicolumn{2}{|c|}{ A Detector } & \multicolumn{2}{|c|}{ B Detector } \\
\hline & Counts $\mathrm{s}^{-1}$ & Live Time $(s)^{b}$ & Counts $\mathrm{s}^{-1}$ & Live Time (s) \\
\hline $1990 \operatorname{Dec} 8(6.6) \ldots \ldots$ & Total 10.3 & 1000 & Total 6.4 & 1000 \\
\hline $1990 \operatorname{Dec} 8 \ldots \ldots \ldots \ldots$ & A0 6.6 & $\ldots$ & B0 2.7 & $\ldots$ \\
\hline $1990 \operatorname{Dec} 8 \ldots \ldots \ldots$ & A1 2.1 & $\ldots$ & B1 0.3 & $\ldots$ \\
\hline $1990 \operatorname{Dec} 8 \ldots \ldots \ldots \ldots$ & A2 0.6 & $\ldots$ & B2 0.3 & $\ldots$ \\
\hline $1990 \operatorname{Dec} 8 \ldots \ldots \ldots \ldots$ & A3 0.3 & $\begin{array}{l}\cdots \\
\ldots\end{array}$ & B3 2.3 & $\ldots$ \\
\hline $1990 \operatorname{Dec} 8 \ldots \ldots \ldots \ldots$ & A4 0.7 & $\ldots$ & B4 0.8 & $\ldots$ \\
\hline $1990 \operatorname{Dec} 9(7.6) \ldots \ldots$ & Total 10.5 & 200 & Total 9.9 & 200 \\
\hline $1990 \operatorname{Dec} 9 \ldots \ldots \ldots \ldots$ & A0 0.8 & $\ldots$ & B0 0.3 & $\ldots$ \\
\hline $1990 \operatorname{Dec} 9 \ldots \ldots \ldots$ & A1 2.3 & $\ldots$ & B1 0.1 & $\ldots$ \\
\hline $1990 \operatorname{Dec} 9 \ldots \ldots \ldots$ & A2 7.1 & $\ldots$ & B2 0.1 & $\ldots$ \\
\hline $1990 \operatorname{Dec} 9 \ldots \ldots \ldots \ldots$ & A3 0.2 & $\ldots$ & B3 1.4 & $\ldots$ \\
\hline $1990 \operatorname{Dec} 9 \ldots \ldots \ldots$ & A4 0.1 & $\ldots$ & B4 8.0 & $\ldots$ \\
\hline
\end{tabular}

${ }^{\text {a }}$ BBXRT MET shown in parentheses. MET $0.0=$ JD 2,448,227.784039.

${ }^{b}$ Live times are the same for all pixels of a particular observation. 


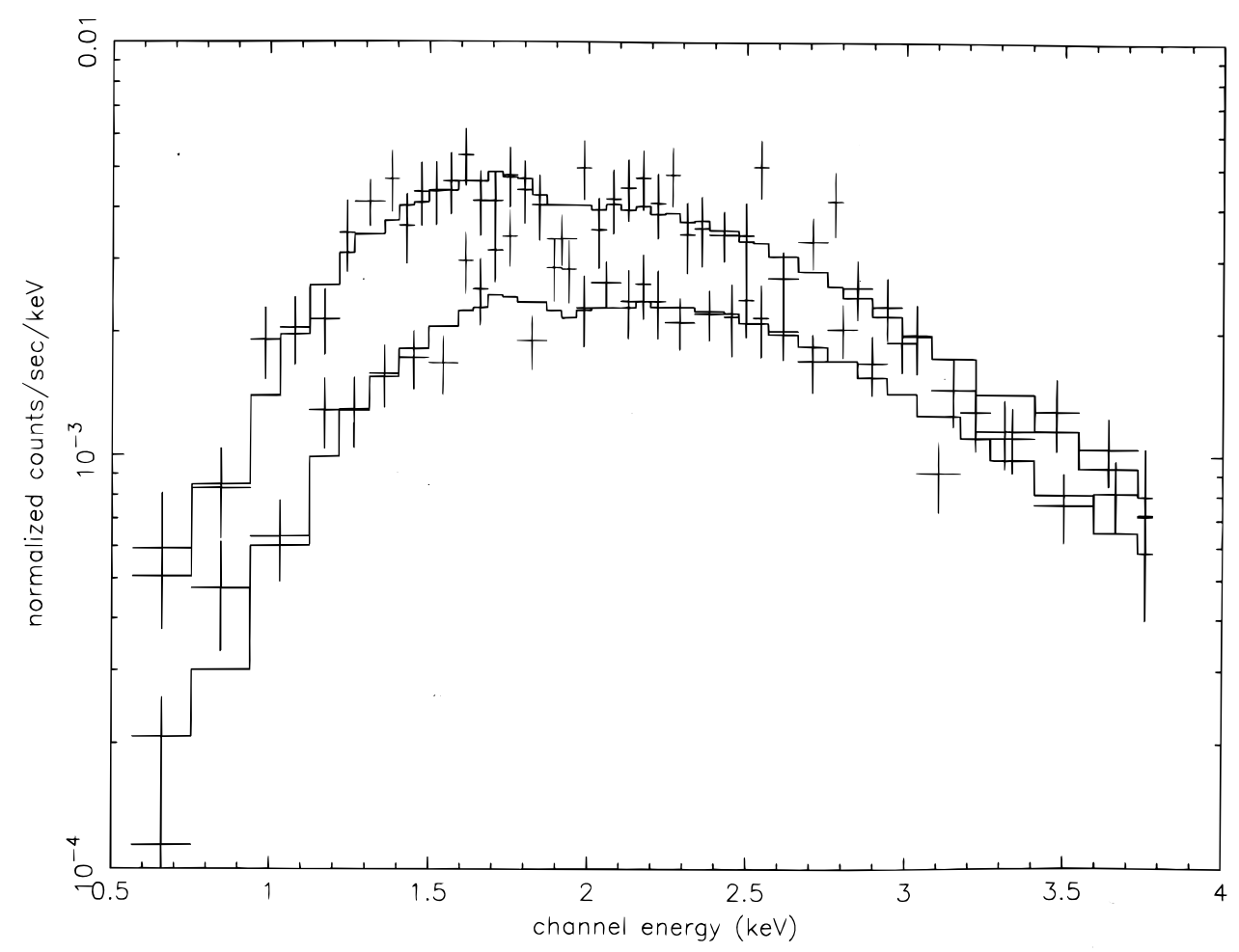

FIG. 2.-Comparison of sample SSS spectra from the 1979 May 11 observation with best fitting BB + PL models shown. The MPC was used in determining the fit, but is omitted for ease of presentation.

which two spectra were accumulated per spacecraft orbit to monitor changes in spectral parameters as a function of phase and intensity. The corresponding backgroundsubtracted MPC data were accumulated in $40.96 \mathrm{~s}$ bins (Arnaud, K. 1996, private communication). The SSS background was not accumulated simultaneously and consequently is modeled from $10 \mathrm{ks}$ of blank sky observations. The background consists of two components: a component related to cosmic-ray and particle events and almost independent of energy, and a second, more variable component above $3 \mathrm{keV}$ (Szymkowiak 1985; Christian 1993). Total background rates were generally small $\left(<0.2\right.$ counts $\mathrm{s}^{-1}$ in the energy range of interest), and the background correction factor for the second component was set to 0.5 .

BBXRT spectra were accumulated for good time intervals allowed by the five anticoincidence flags (Weaver et al. 1995). The BBXRT background was obtained from scaling the internal background during observations to the closed cover internal background. This excess is mainly the result of particle events (Weaver et al. 1995)

Spectral fitting was performed with the XSPEC package (Shafer, Arnaud, \& Haberl 1989). The responses used for the SSS and MPC data were those generated for the survey of LMXB with this same data set (Christian 1993; Christian \& Swank 1997).

The most current BBXRT responses were used (Weaver 1994; Weaver et al. 1995). No aspect solutions were available for the BBXRT observations, and on-axis responses were used. The first BBXRT observation had an almost Gaussian distribution of counts among the pixels, which is expected for the source centered in the central pixel. Spectral fits made with responses generated assuming the pointings were $2^{\prime}$ off center were not substantially different. The second BBXRT observation of December 9 was centered in the outer pixels, A2 and B4. For the December 8 obser- vation we simultaneously fitted the four BBXRT pixels with the most counts to obtain the best determination of the model parameters.

\subsection{Models}

Spectral models often used in the description of LMXB data were fitted to the spectra of M15. These models attempt to represent a physical model, but at least provide a phenomenological description in which to compare different source states, different observations, and other sources. Forms of these continuum models include power-law (PL), blackbody (BB), optically thin thermal bremsstrahlung (TB) with Gaunt factor compiled by Kellogg, Baldwin, \& Koch (1975), and a physically thin but optically thick accretion disk model (DBB) (Mitsuda 1984). Additionally, a model of the form constant $\times E^{-\Gamma} \exp (-E / k T)$, which is called a "Wien-Boltzmann" form because of its ability to mimic both spectral forms and describe an approximation to unsaturated Comptonization (White, Peacock, \& Taylor 1985), was also fitted to the data. For historical reasons we denote it USC. The Sunyaev \& Titarchuk (1980) form for the Comptonization of soft photons scattering off hot electrons was also fitted (CompST). Two-component models were constructed from the simple continuum models, often including one blackbody. All spectral models include absorption by material along the line of sight, exp $\left[-\sigma(E) N_{\mathrm{H}}\right]$, where $\sigma(E)$ is the photoelectric cross section, taken from work by Morrison \& McCammon (1983). The XSPEC "varabs" model was used to discriminate among the absorption contributions of different metals.

\section{SPECTRAL RESULTS}

The 0.5-20.0 keV SSS and MPC spectra were fitted with the aforementioned continuum models. Generally, USC was the best-fitting single-component model. The spectral 
index of the USC model, $\Gamma$, ranged from -0.7 to 1.0 for the lowest luminosity phases, and from 0.9 to 1.3 for the higher luminosity phases. The DBB model gave similar $\chi^{2}$ for the early (1978 December 5) observations with reasonable mass accretion rates of one-hundredth of Eddington. A PL model did as well as the USC for the low column observations of May 10 that spanned phases $0.2-0.3$. Two-component models, such as BB + PL and BB + TB, gave $\chi^{2}$ slightly better than the USC model with blackbody temperatures of $1 \mathrm{keV}$. TB was equivalent to USC for the BBXRT observations, and the addition of a blackbody was not significant. Sample SSS spectra are shown in Figure 2. Table 3 presents sample results of spectral fits to the SSS/MPC and BBXRT data.

\subsection{Model Parameters as a Function of Phase}

$\mathrm{BB}+\mathrm{PL}$ was found to be the best-fitting model to EXOSAT medium-energy (ME) observations (Callanan et al. 1987), and was used to fit for spectral changes as a function of luminosity and phase. The BB + PL model may better represent a simplified physical model (discussed below). Spectral parameters plotted as a function of phase and luminosity are shown in Figures 3 and 4, respectively. Most notable is the correlation of $N_{\mathrm{H}}$ with phase. The hydrogen column, $N_{\mathrm{H}}$, increases an order of magnitude from its minimal values set from interstellar reddening $\left(N_{\mathrm{H}}=7 \times 10^{20} \mathrm{~cm}^{-2}\right.$; van Paradijs et al. 1990 ; see also Naylor et al. 1992) at phases $0.0-0.3$ to $10^{22} \mathrm{~cm}^{-2}$ at phase $0.4-0.7$. There is no obvious correlation between column density and luminosity. The highest luminosity spectra have the least column, suggesting that the column density is anticorrelated with luminosity. This is similar to results of Hertz \& Grindlay (1983), who observed an inverse correlation between the column density and luminosity for a different set of Einstein MPC observations. Our results are in disagreement with Callanan et al. (1987), who observed

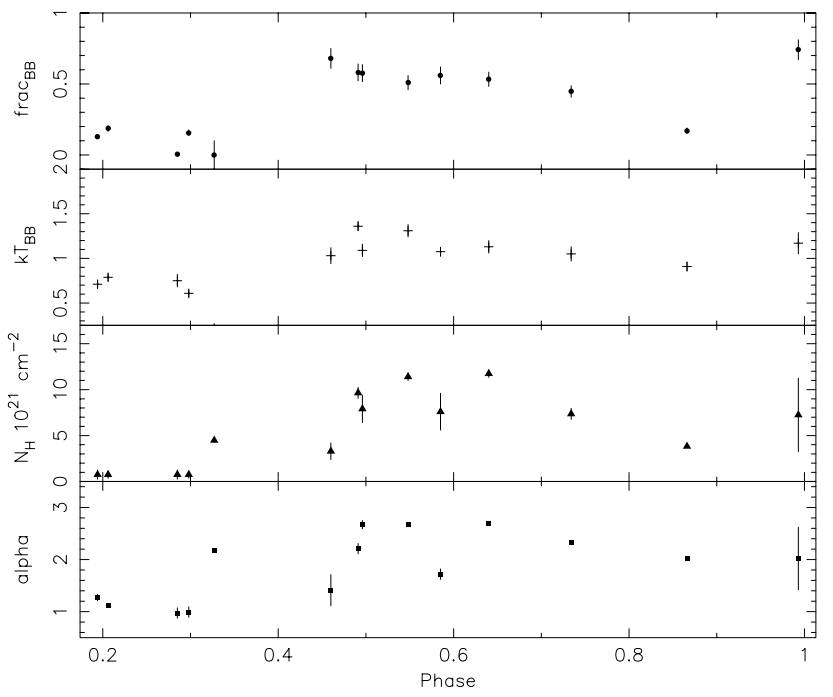

FIG. 3.-BB + PL spectral parameters plotted as a function of phase. Top panel is the fractional contribution of the BB in the BB + PL model, followed by the $\mathrm{BB}$ temperature in $\mathrm{keV}$, the column density in units of $10^{21}$ $\mathrm{cm}^{-2}$, and the spectral index of the power-law model, $\alpha$.

the column density to be correlated with the luminosity using observations obtained with the EXOSAT ME instrument.

\subsection{Metallicity}

Although the moderate resolution of BBXRT does not allow us to make a direct measure of the contributions from individual elements, we performed several spectral fitting tests to constrain the global abundances of metals. We performed these tests with the aforementioned continuum model, but present the results for the best-fitting continuum model, TB. The BBXRT data did not require a BB com-

TABLE 3

SAMPLE ONE-COMPONENT Fit Parameters

\begin{tabular}{|c|c|c|c|c|c|}
\hline Model & Norm & $k T$ & $\alpha / \Gamma^{\mathrm{a}}$ & $N_{\mathrm{H}}$ & $\chi^{2} /$ dof \\
\hline \multicolumn{6}{|c|}{ SSS/MPC: 11 May 1979 (D1) } \\
\hline PL & $0.17 \pm 0.01$ & & $2.69 \pm 0.05$ & $18 \pm 1$ & $215 / 75$ \\
\hline TB & $0.036 \pm 0.002$ & $4.1 \pm 0.2$ & $\ldots$ & $9.4 \pm 0.7$ & $122 / 75$ \\
\hline BB $\ldots$ & $0.01 \pm 0.001$ & $1.10 \pm 0.01$ & & 0.7 & $243 / 75$ \\
\hline DBB . & $0.015 \pm 0.002$ & & $1.66 \pm 0.03$ & $4.3 \pm 0.5$ & $90 / 75$ \\
\hline USC $\ldots \ldots \ldots \ldots$ & $0.036 \pm 0.002$ & $2.0 \pm 0.6$ & $0.06 \pm 0.06$ & $3.5 \pm 0.6$ & $91 / 74$ \\
\hline CompST ...... & $0.032 \pm 0.006$ & $1.3 \pm 0.1$ & $26 \pm 3$ & $0.6 \pm 0.1$ & $82 / 74$ \\
\hline \multicolumn{6}{|c|}{ SSS/MPC: 11 May 1979 (D2) } \\
\hline PL & $0.09 \pm 0.01$ & & $2.35 \pm 0.05$ & $23 \pm 1$ & $127 / 75$ \\
\hline & $0.019 \pm 0.001$ & $5.8 \pm 0.3$ & $\ldots$ & $15 \pm 1$ & $92 / 75$ \\
\hline BB $\ldots \ldots \ldots \ldots$ & $0.006 \pm 0.0006$ & $1.23 \pm 0.01$ & & $\overline{0.7}$ & $143 / 75$ \\
\hline DBB $\ldots \ldots \ldots$ & $0.009 \pm 0.001$ & & $2.02 \pm 0.05$ & $7.8 \pm 0.8$ & $85 / 75$ \\
\hline USC $\ldots \ldots \ldots$ & $0.023 \pm 0.001$ & $2.8 \pm 0.3$ & $0.4 \pm 0.2$ & $8 \pm 1$ & $82 / 74$ \\
\hline CompST ...... & $0.016 \pm 0.004$ & $1.5 \pm 0.1$ & $28 \pm 5$ & $0.8 \pm 0.2$ & $78 / 74$ \\
\hline \multicolumn{6}{|c|}{ BBXRT (4 Pixels) } \\
\hline PL & $0.08 \pm 0.003^{b}$ & & $2.12 \pm 0.03$ & $4.9 \pm 0.2$ & $774 / 682$ \\
\hline & $0.03 \pm 0.001$ & $4.8 \pm 0.2$ & $\ldots$ & $3.1 \pm 0.1$ & $700 / 682$ \\
\hline $\mathrm{BB} \ldots \ldots \ldots \ldots$ & $0.002 \pm 0.00004$ & $0.74 \pm 0.01$ & $\ldots$ & $0.7 \pm 0.3$ & $1401 / 682$ \\
\hline DBB ............ & $0.003 \pm 0.0004$ & & $2.5 \pm 0.2$ & $1.4 \pm 0.1$ & $749 / 682$ \\
\hline USC $\ldots \ldots \ldots \ldots$ & $0.07 \pm 0.003$ & $3.7 \pm 0.4$ & $1.1 \pm 0.1$ & $2.8 \pm 0.3$ & $697 / 681$ \\
\hline CompST ...... & $0.06 \pm 0.002$ & $1.7 \pm 0.1$ & $16.7 \pm 0.9$ & $3.7 \pm 0.2$ & $703 / 681$ \\
\hline
\end{tabular}

${ }^{a}$ Column is $\alpha$ or $\Gamma$ for the PL or USC models, respectively, and the Eddington mass accretion rate or optical depth for the DBB and CompST models, respectively.

${ }^{b}$ Norms are from the BBXRT A0 pixel. 


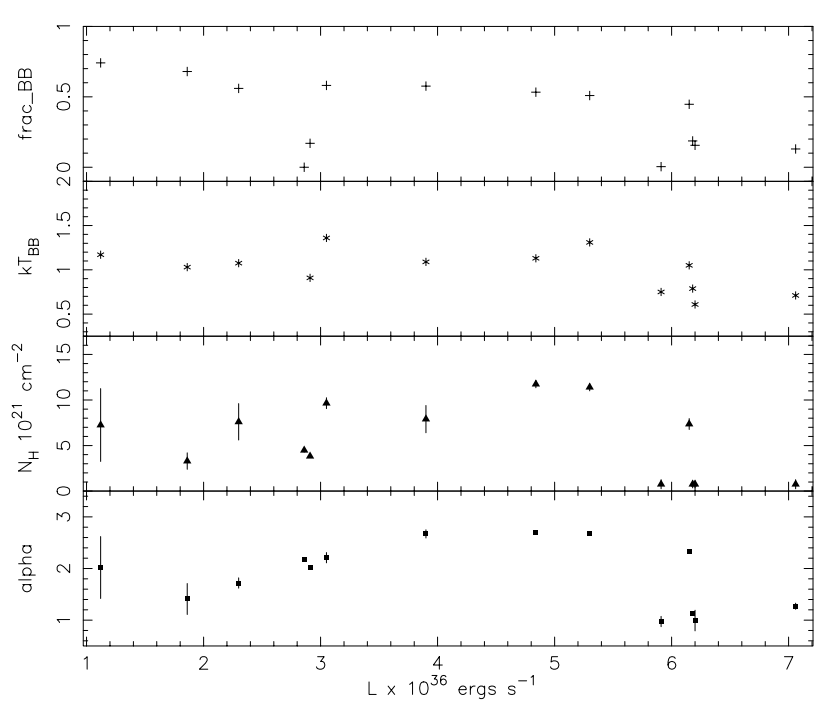

FIG. 4. - BB + PL spectral parameters plotted as a function of luminosity in units of $10^{36} \mathrm{ergs} \mathrm{s}^{-1}$. Panels are the same as in Fig. 3.

ponent. First, we tested for metals BBXRT is expected to be sensitive to: possibly $\mathrm{O}$ with a neutral edge energy of 0.53 $\mathrm{keV}$ and at the edge of the bandpass, and $\mathrm{Fe}$ and $\mathrm{Si}$, which have neutral edge energies of $0.71(\mathrm{Fe} \mathrm{L})$ and $1.84 \mathrm{keV}$, respectively. We took advantage of the capabilities of the XSPEC varabs model and linked $\mathrm{H}, \mathrm{He}, \mathrm{C}, \mathrm{N}, \mathrm{Ne}, \mathrm{Na}, \mathrm{Mg}$, $\mathrm{Al}, \mathrm{S}, \mathrm{Cl}, \mathrm{Ar}, \mathrm{Ca}, \mathrm{Cr}, \mathrm{Ni}$, and $\mathrm{Co}$ as one fit parameter, but left $\mathrm{O}, \mathrm{Fe}$, and $\mathrm{Si}$ as free parameters. We found the $\mathrm{O}$ and $\mathrm{Si}$ poorly determined, with $\mathrm{Fe}$ nearly consistent with the solar abundance: $(6.5 \pm 1.3) \times 10^{17} \mathrm{~cm}^{-2}$.

Second, we took advantage of the capabilities of the XSPEC varabs model to combine the effects of individual metals. We constructed a model which grouped $\mathrm{H}$ and $\mathrm{He}$ as one fit parameter- $N(\mathrm{H}, \mathrm{He})$ - and $\mathrm{C}, \mathrm{N}, \mathrm{O}, \mathrm{Ne}, \mathrm{Na}, \mathrm{Mg}$, $\mathrm{Al}, \mathrm{Si}, \mathrm{S}, \mathrm{Cl}, \mathrm{Ar}, \mathrm{Ca}, \mathrm{Cr}, \mathrm{Fe}, \mathrm{Ni}$, and $\mathrm{Co}$ as another parameter- $N$ (metals). The varabs model uses the solar abundance of metals. The four detector pixels with the most counts were fitted simultaneously with a TB continuum model multiplied by the varabs model and also a separate, fixed absorption for interstellar material, set by $E_{B-V}$.

To test the hypothesis that X2127+119 has the same abundance as the host cluster, we fixed the $N$ (metals) parameter to $1 \%$ of its solar value relative to $N(\mathrm{H}, \mathrm{He})$. [Metallicities for M15 in the literature range from log $(\mathrm{Fe} / \mathrm{H})=-2.15(\mathrm{Zinn} \&$ West 1984) to $\log (\mathrm{Fe} / \mathrm{H})=$ -1.76 (Pilachowski, Sneden, \& Wallerstein 1983), and therefore we adopted a value of -2 for ease of use.] This model increased $N(\mathrm{H}, \mathrm{He})$ to $(6.6 \pm 0.4) \times 10^{21} \mathrm{~cm}^{-2}$ while increasing $\chi^{2}$ by 26 . Applying the $F$-test (Bevington 1969) to this result gives a probability $\leq 0.001$ of being random. We show the TB and TB*varabs model fits and residuals in Figures $5 a-5 c$. Repeating this fitting procedure for other forms of the continuum generally gave much larger increases in $\chi^{2}$, with the exception of the USC model which had an increase in $\chi^{2}$ of 10 . Similarly, applying the $F$ test to the USC continuum gives a probability $\leq 0.002$ of being random. The additional free parameter of the USC model, $\Gamma$, makes it possible to adjust the low energies, and the result is still inconsistent, but is slightly more consistent than TB with the noted cluster metallicity.

Allowing the $N(\mathrm{H}, \mathrm{He})$ and $N($ metals $)$ parameters to be free had the result of placing all of the absorption in the metals with $N(\mathrm{H}, \mathrm{He})=0.0_{-0.0}^{+0.097} \times 10^{21} \mathrm{~cm}^{-2}$ and $N$ (metals) $=1.25_{-0.2}^{+0.03} \times 10^{18} \mathrm{~cm}^{-2}$. A confidence contour of the $N(\mathrm{H}, \mathrm{He}$ ) and $N$ (metals) parameters shows them to be anticorrelated. Perhaps this is not a real physical effect but a fitting artifact caused by the fact that $\mathrm{C}$ and $\mathrm{N}$ edges are below the bandpass and are unconstrained. Alternatively, the X-ray implication of a solar-like abundance of metals may be consistent with the fact that measurements of $C, N$, and O (Kraft 1994) and O (Minniti et al. 1996) have shown them to be overabundant as compared to Fe in M15.

\subsection{Line Emission}

Narrow-line emission at $6.4 \mathrm{keV}$ is expected from fluorescence of cool $\mathrm{Fe}$ in a corona, and broad lines between 6.7 and $6.9 \mathrm{keV}$ may be formed from the photoionizationrecombination of hydrogenic and He-like iron in a hot corona. No strong emission lines were detected in the BBXRT spectra. A narrow emission line near $6.4 \mathrm{keV}$ was evident in the second BBXRT observation. It could be best fitted as a narrow line at $6.44 \pm 0.06 \mathrm{keV}$ with a $\sim 225 \mathrm{eV}$ equivalent width. However, it is not statistically significant. A $3 \sigma$ upper limit to a narrow emission line at $6.4 \mathrm{keV}$ in the first BBXRT observation was $150 \mathrm{eV}$. We found a $3 \sigma$ upper limit of $110 \mathrm{eV}$ to a broad line at $6.7 \mathrm{keV}$ with a width of 300 $\mathrm{eV}$. Line emission from Fe $\mathrm{L}$ was found in several LMXBs (Vrtilek et al. 1991). A $3 \sigma$ upper limit to a line at $0.77 \mathrm{keV}$ (possibly from O VIII or Fe XVIII; Vrtilek et al. 1991) was found to be $33 \mathrm{eV}$ for the BBXRT spectrum and $\sim 100 \mathrm{eV}$ for individual SSS spectra.

\section{DISCUSSION}

The complex optical and X-ray behavior observed from $\mathrm{X} 2127+119$ has made it difficult to define a physically consistent model. Observational clues, such as its optical and $\mathrm{X}$-ray light curves and X-ray to optical luminosity ratio of 20 , support the accretion disk corona interpretation for this system, but the newly detected X-ray burst limits the extent of the corona. The moderate resolution and limited bandpasses of both the SSS and the BBXRT limit the complexity of testable spectral models. We have used a BB + PL model as more a phenomenological description than a physical model. It may be liberally interpreted in terms of a physical model in which the BB originates in an optically thick region close to the neutron star, and the power law is formed by scattering of the BB or soft photons in an extended region above the accretion disk. The $\mathrm{BB}$ radii derived from the fits, typically $\sim 2-5 \mathrm{~km}$ for a distance of 10 $\mathrm{kpc}$, are too small to represent the entire surface component of the neutron star, although the detection of the burst proves it is visible. Rather, it is probably the optically thick outer portion of the accretion disk, but the SSS data are limited in distinguishing between a disk model and a BB because of the unknown column density and lack of lowenergy effective area.

Although X2127+ 119 falls into the Atoll LMXB classification, two-component spectral models are generally found for the Z LMXBs (Schulz, Hasinger, \& Trumper 1989). The variations of the BB + PL model parameters with source intensity seen here are very different from variations of similar two-component models of the Z LMXBs. The BB fraction, temperature, and radius usually increase with luminosity for the bright $\mathrm{Z}$ source. However, for $\mathrm{X} 2127+119$ the BB fraction, temperatures, and radii are smallest as the source approaches maximum luminosity (see 

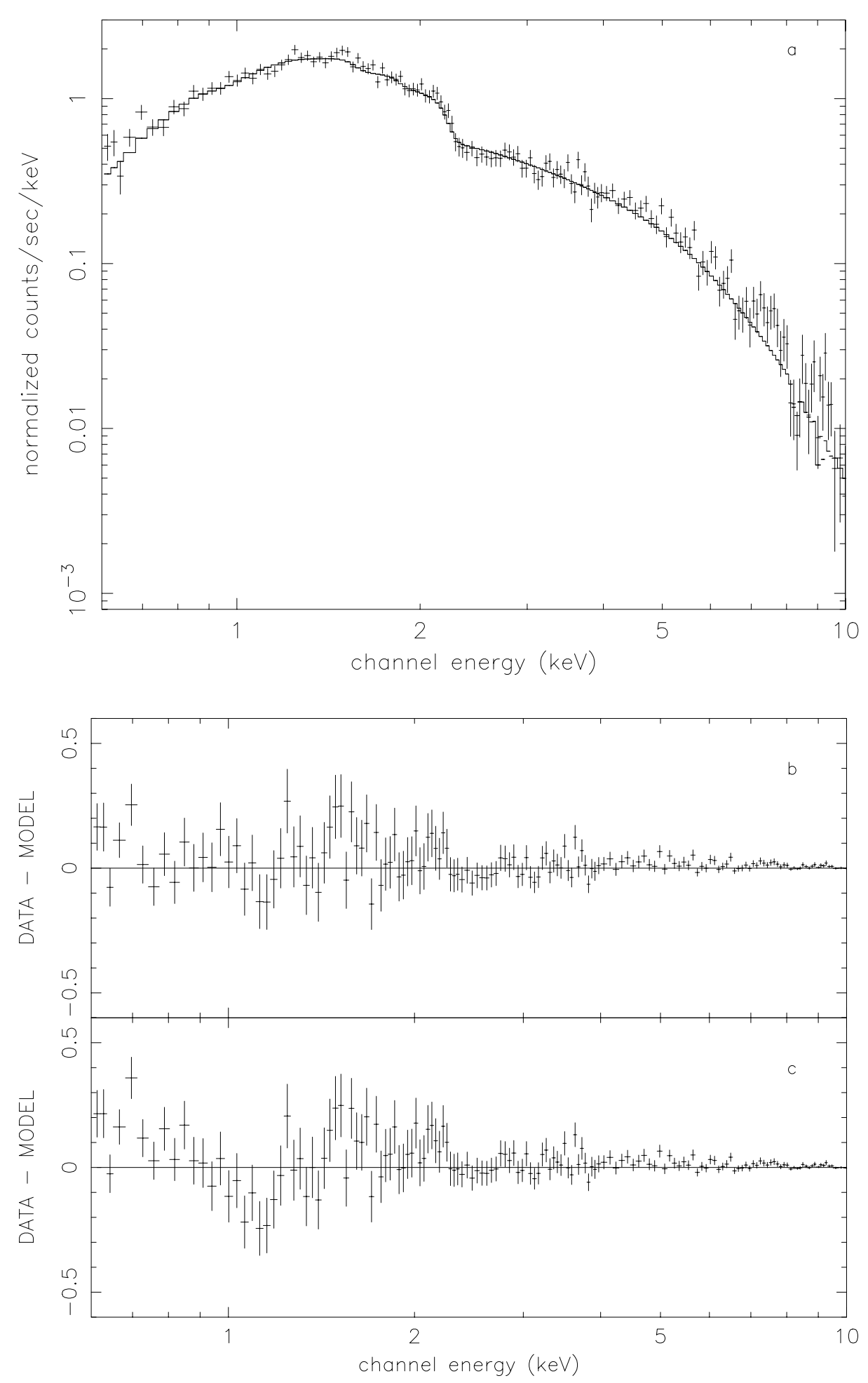

Fig. 5.-Shown are data from 1978 December 9 observation of X2129+117. The fitting was performed with the 4 pixels with the most counts, which are grouped together for ease of presentation. Shown are $(a)$ the data and TB continuum model, $(b)$ the residuals to that model, $(c)$ the residuals to the TB*varabs model fitted with the $N$ (metals) set at one-hundredth of $N(\mathrm{H}, \mathrm{He})$ (see text).

Fig. 4). The power-law spectral index decreases from being steep $(\sim 2.5$, spectrally soft $)$ to being more flat $(<1$, spectrally hard) with increasing luminosity. However, there is an early (day 339) observation which has high luminosity with $\alpha$ of 2.3, and two low-luminosity observations where $\alpha<2$. Although we are limited by sampling, it appears the source has different intensity states independent of the orbital phase. This has been seen with the difficulty in detecting the $17.1 \mathrm{hr}$ modulation in the X-ray data, as in the case for the EXOSAT ME data (Callanan et al. 1987).

The most notable correlation with phase presented here is the order-of-magnitude increase in column density observed between phases 0.4 and 0.7 (see Fig. 3). The energy dependence of this change is easily noted in a hardness ratio 
diagram, which does not depend on the chosen spectral model. Therefore the increase in photoelectric absorption is the result of the increase in column. This increase can be easily explained if we are viewing the source through the extended outer edge of the accretion disk rim, such as would be expected where the accretion stream interacts with the disk. However, near optical phase 0.5 the neutron star is eclipsing the companion, and the accretion stream and disk bulge are behind the neutron star. We can solve this problem by simply arguing that the optical phase 0.0 is wrongly defined, i.e., at phase 0.0 the companion is being eclipsed by the neutron star, and that at phase 0.5 (when the neutron star is eclipsed by the companion) we are seeing the $\mathrm{X}$-ray flux through the accretion bulge of the disk. Perhaps a more plausible argument is that the optical phase is correct, and the high column observed between phases 0.5 and 0.7 is similar to the secondary minimum originally mistaken for the orbital period (Callanan 1993). This extra absorption could come from material that flows over the top of the neutron star as discussed by Frank, King, \& Lasota (1987). The system does show a variety of behavior in the optical between phases 0.2 and 0.7 , and Ilovaisky et al. 1993 argue for obscuring material along the line of sight near optical and X-ray phases 0.5 .

We can then explain the lack of increase in column near phase 0.2 shown here as the result of variations in the extent of the corona. Radiation from the central source may cause the outer disk and the disk bulge to increase in vertical extent to form an accretion disk corona (White \& Holt 1982), that would vary as the system geometry changes with phase. Ginga observations (presented by Ilovaisky et al. 1993; Callanan 1993) show an increase in column near phase 0.0 . The BBXRT observation at phase 0.3 had a much larger column than the SSS and MPC observations spanning phases $0.2-0.29$. If we interpret the X-ray behavior of $\mathrm{X} 2127+119$ as the bulge in the accretion disk, it requires the assumption that the extent of the bulge was much smaller for phases $0.2-0.29$ during the SSS and MPC observations presented here. Perhaps this is reasonable based on the burst detection (van Paradijs et al. 1990) that shows the corona cannot be as extensive as other ADC sources such as X1822-371, and that its thickness has been observed to vary.

The BBXRT spectra were used to distinguish between the contribution of $\mathrm{H}$ and $\mathrm{He}$ and metals in the absorbing column. The fitting results are generally inconsistent with the overall cluster metallicity of $1 \%$ of solar, although complex spectral models can be more consistent with the cluster metallicity. The discrepancy observed for the preferred forms of the continuum is puzzling for an extremely metal-poor cluster $[\log (\mathrm{Fe} / \mathrm{H})=-2]$ such as M15. One possibility is that the moderate resolution of BBXRT cannot uniquely distinguish the metallic absorption edges, but the large increase in $\chi^{2}$ for the underabundant model argues against this. A second possibility is that the source has a large abundance of metals when compared to the cluster. $\mathrm{C}, \mathrm{N}$, and $\mathrm{O}$ have been observed to be enhanced relative to Fe for M15 (Minniti et al. 1996; Kraft 1994). Also, X-ray measurements have found abundances greater than solar for Cyg X-1 (Kitamoto et al. 1984; Vrtilek et al. 1991) and Sco X-1 (Vrtilek et al. 1991), and for the dipping system X0748-676 (Parmar et al. 1986). This may be the result of CNO burning on the surface of the neutron star or the result of nonstandard evolution for this object.

Future high-resolution X-ray observations that can better resolve individual contributions of metals will place tighter constraints on abundances in X2117+119. Similar high-resolution observations that could monitor possible iron-line emission as a function of phase will better constrain the accretion disk coronal model of X2127+119.

D. C. acknowledges partial support of this research though a NASA Astrophysical Data Products grant. We thank M. Garcia for useful discussions on X2127 + 119. We also thank Paul Callanan for critical review of the manuscript and suggested improvements.

\section{REFERENCES}

Aurière, M., Le Fèvre, O., \& Terzan, A. 1984, A\&A, 138, 415

Bevington, P. R. 1969, Data Reduction and Error Analysis in the Physical Sciences (New York: McGraw-Hill)

Callanan, P. J. 1993, PASP, 105, 961

Callanan, P. J., Fabian, A. C., Tennant, A. F., Redfern, R. M., \& Shafer, R. A. 1987, MNRAS, 224, 781

Christian, D. J. 1993, Ph.D. thesis, Univ. Maryland

Christian, D. J., \& Swank, J. H. 1997, ApJS, in press

Dotani, T., et al. 1990, Nature, 347, 534

Frank, J., King, A. R., \& Lasota, J.-P. 1987, A\&A, 178, 137

Gaillardetz, R., Bjorkholm, P., Mastronardi, R., Vanderhill, M., \& Howland, D. 1978, IEEE Trans. Nucl. Sci., NS-25, 437

Giacconi, R., et al. 1979, ApJ, 230, 540

Hertz, P. 1987, ApJ, 315, L119

Hertz, P., \& Grindlay, J. E. 1983, ApJ, 275, 105

Ilovaisky, S. A., Aurière, M., Koch-Miramond, L., Chevalier, C., Cordoni, J.-P., \& Crowe, R. A. 1993, A\&A, 270, 139

Joyce, R. M., Becker, R. H., Birsa, F. B., Holt, S. S., \& Noordzy, M. P. 1978, IEEE Trans. Nucl. Sci., NS-25, 453

Kellogg, E., Baldwin, J. R., \& Koch, D. 1975, ApJ, 199, 299

Kitamoto, S., et al. 1984, PASJ, 36, 731
Kraft, R. P. 1994, PASP, 106, 553

Minniti, D., Peterson, R. C., Geisler, D., \& Clariá, J. J. 1996, AJ, in press Mitsuda, K. 1984, Ph.D. thesis, Univ. Tokyo

Morrison, R., \& McCammon, D. 1983, ApJ, 270, 119

Naylor, T., Charles, P. A., Hassall, B. J. M., Raymond, J. C., \& Nassiopoulos, G. 1992, MNRAS, 255, 1

Parmar, A. N., White, N. E., Giommi, P., \& Gottwald, M. 1986, ApJ, 308, 199

Pilachowski, C. A., Sneden, C. \& Wallerstein, G. 1983, ApJS, 52, 241

Schulz, N. E., Hasinger, G., \& Trumper, J. 1989, A\&A, 225, 48

Shafer, R. A., Arnaud, K. A., \& Haberl, F. 1989, XSPEC Users Manual

Sunyaev, R. A., \& Titarchuk, L. G. 1980, Soviet Astron. Lett., 12, 117

Szymkowiak, A. E. 1985, Ph.D. thesis, Univ. Maryland

van Paradijs, J., et al. 1990, PASJ, 42, 633

Vrtilek, S. D., et al. 1991, ApJS, 76, 1127

Weaver, K. A. 1994, Ph.D. thesis, Univ. Maryland

Weaver, K. A., et al. 1995, ApJS, 96, 303

White, N. E., \& Holt, S. S. 1982, ApJ, 257, 318

White, N. E., Peacock, A., \& Taylor, B. G. 1985, ApJ, 296, 475

Zinn, R., \& West, M. J. 1984, ApJS, 55, 45 